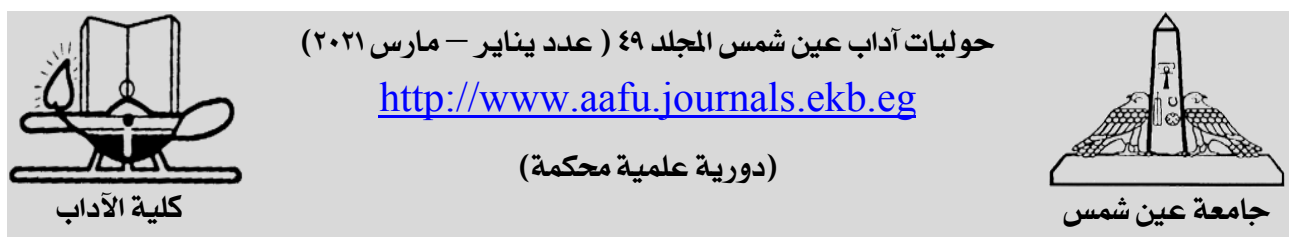

\title{
الأمية المعلوماتية والوباء المعلوماتي في ظل جائحة كورونا الأسباب وطرق كبح هذا الوباء
}

\section{| (سماء الوريكات}

* قسم ادارة المكتبات والمعلومات - كلية السلط للعلوم الانسانية- جامعة البلقاء التطبيقية -الاردن asmaw@bau.edu.jo

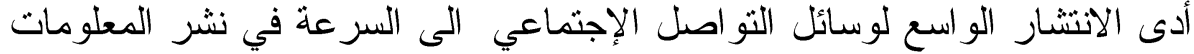

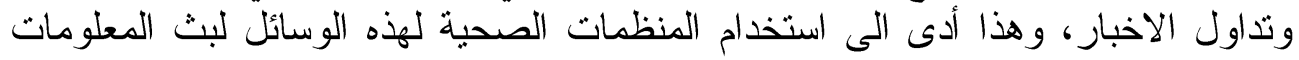

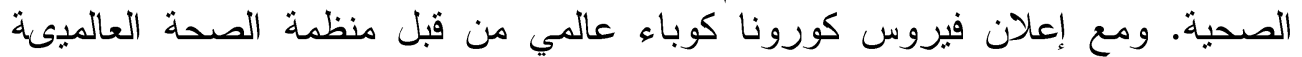

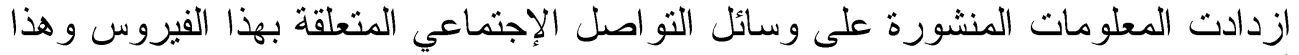

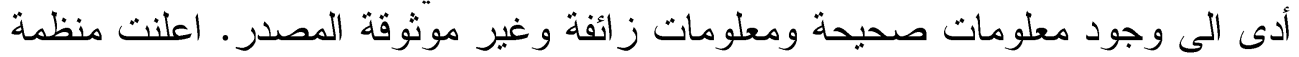

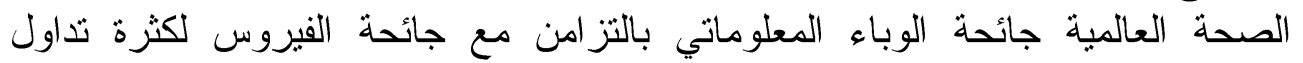
الإخبار المزيفة. يهدف هذا البحث الى التعرف على الوباء المعاء المعلوماتي المرافي افق لجائحة

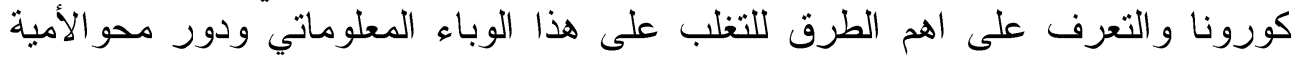

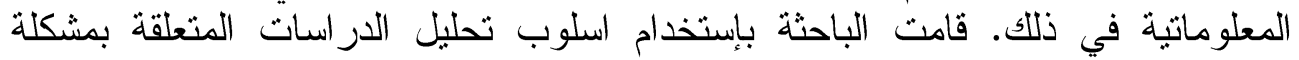

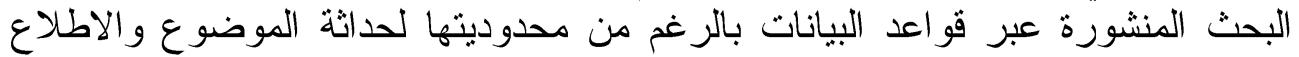

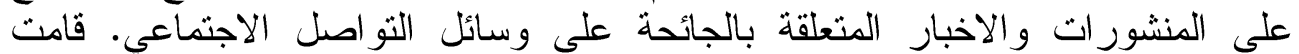

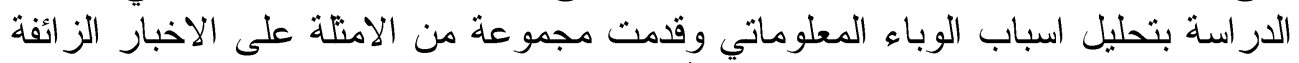

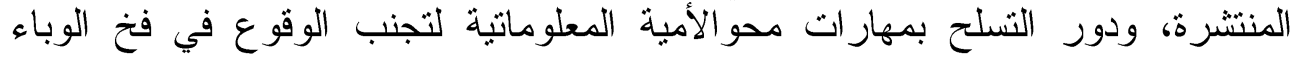

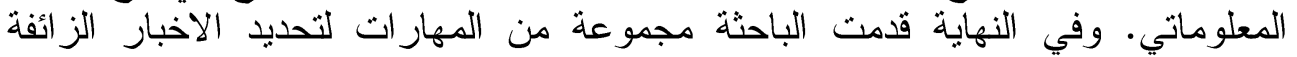
ومجمو عة من التوصيات.

الكلمات الافتاحية: الوباء المعلوماتي، المعلومات الزائفة، سلوك البحث عن

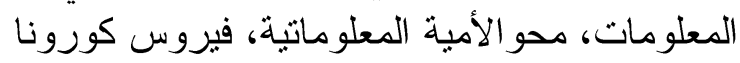




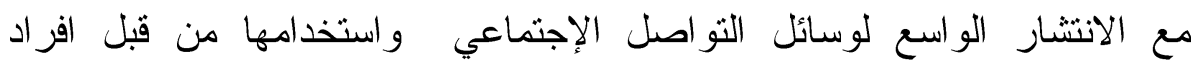
المجتمعات المختلفة، اخذت المنظمات الصحية ببث ونشر وتحميل المعلومات الصحية

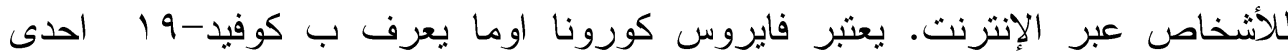

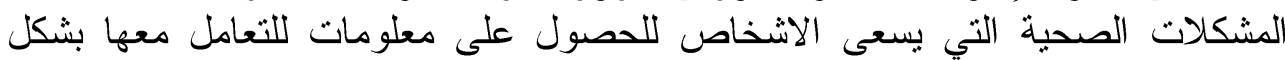

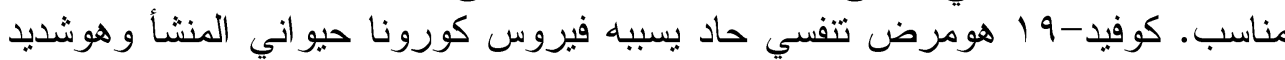
العدوى ويؤثز على حركة المجتمعات و الحياة الإجتماعية اليومية (منظمة الصحة

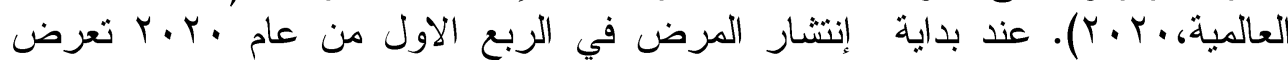

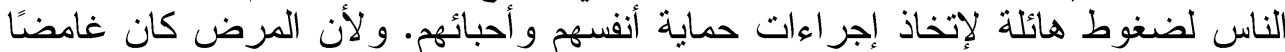
وقت إنتشاره فقد حرص الناس على قر اعة معلومات عن الفيروس لمعرفة طبيعته وكيفية تتجنب الإصابة بالفيروس. هناك كميات هائلة من المعلومات غير المؤكدة حول مختلف جو انب مرض فيروس

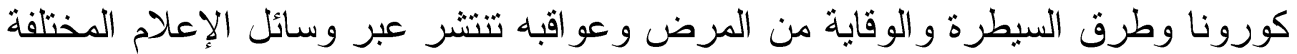

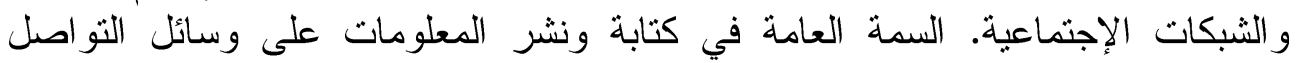

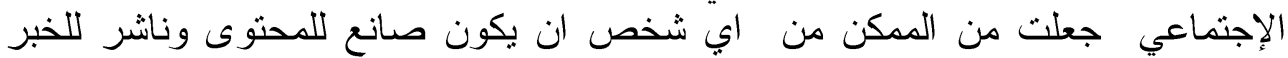

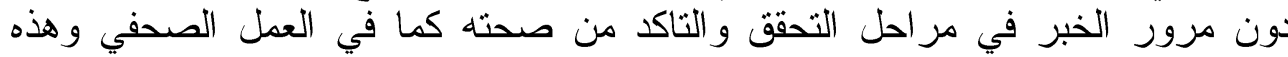

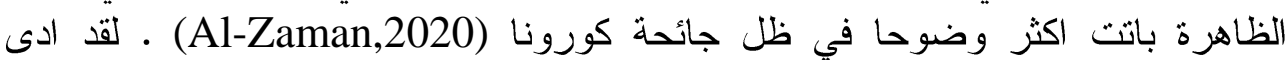
إنتشار الاخبار الكاذبة والمغلوطة الى عودة ظهور مصطيلح جديد وصف بالى بالوباء

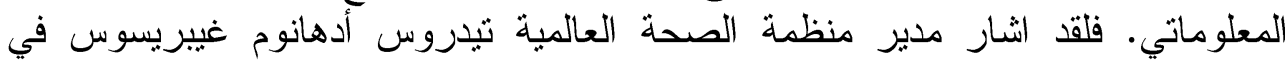

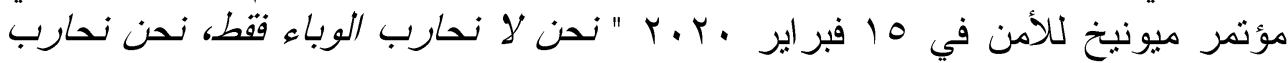

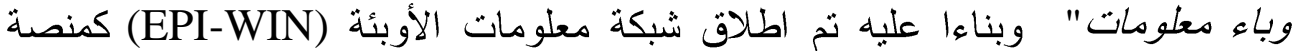

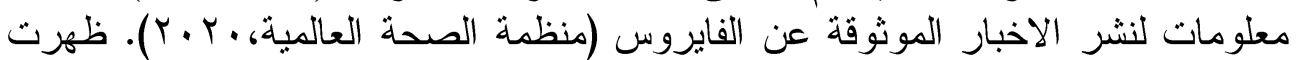

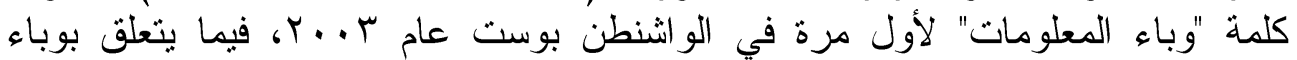
سارس. حيث تم استخدامها لوصف كيف أن بعض الحقائق الممزوجة بالخوف و التكهنات

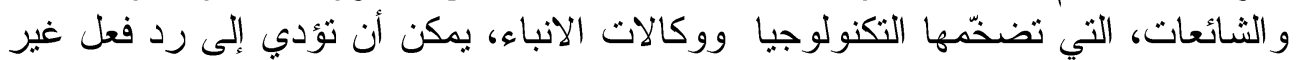

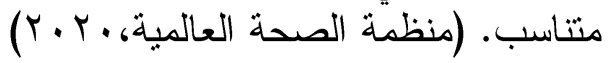
لقد تم تعريف الوباء المعلوماتي Infodemic من قبل منظمة الصحة الصنة العالمية

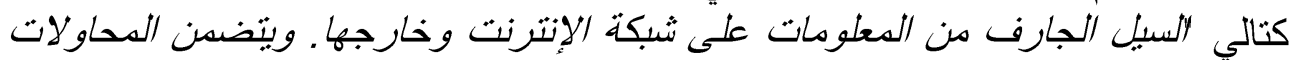

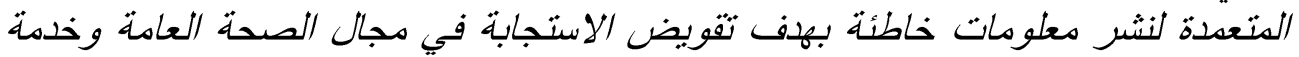

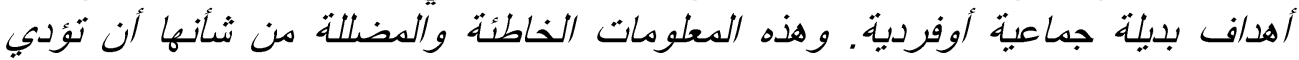

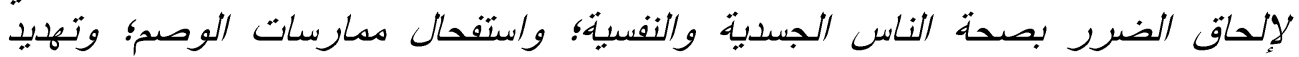

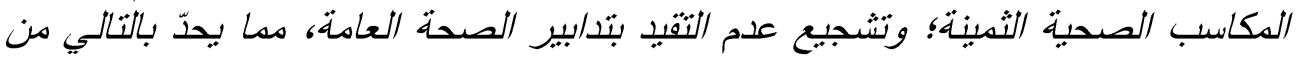

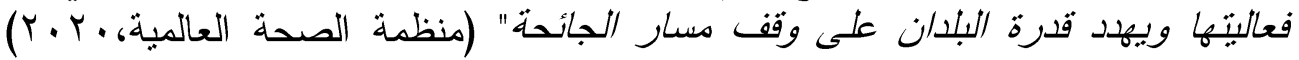

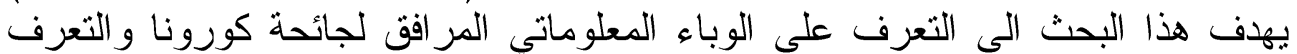

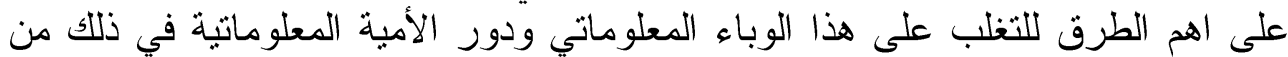

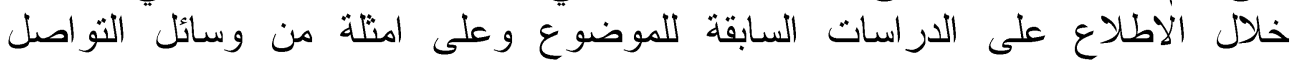
الإجتماعي الاطل 
تحديد اهمية المصطلح الجديد "الوباء المعلوماتي" وربطه بمحو الأمية المعلوماتية

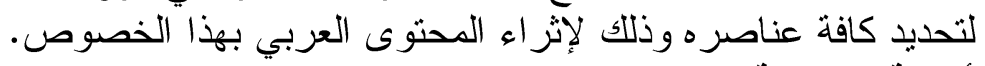
أهمية الار اسلة عناصة

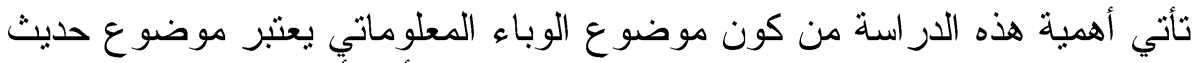

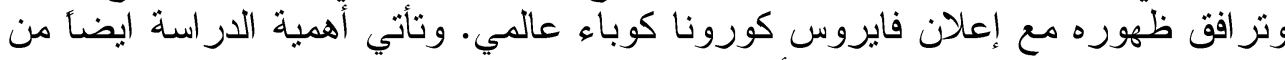
كونها نوضح الر ابط مابين محو الأمية المعلومانية و الوباء المباء الملوماتي وقدرته على كبح جماح هذا الوباء.

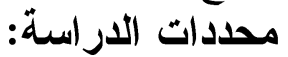

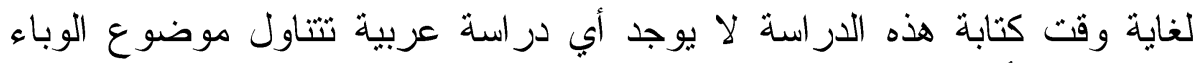

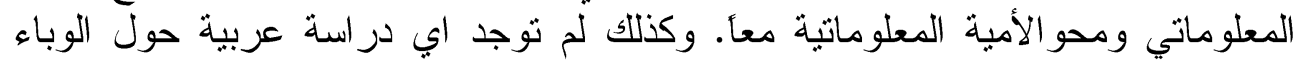

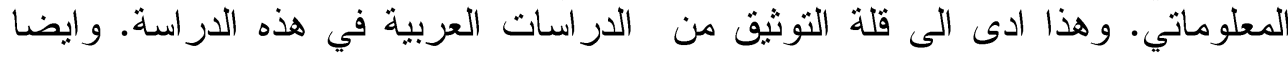

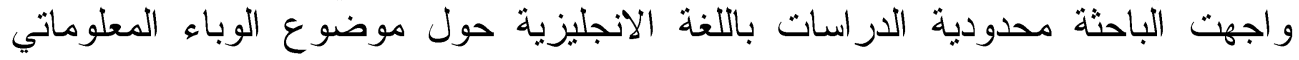
كونه موضو ع عديث. سلوك البحث عن المعلومات الصحية في بدابة الحديث عن مصطلح الوماث الوباء المعلوماتي لابد بداية من التطرق الى مفهوم

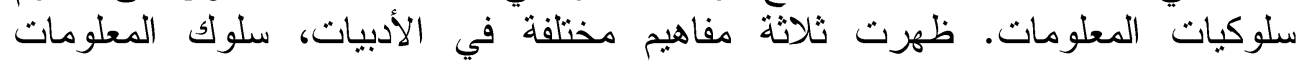
Information ( Information Behaviour)

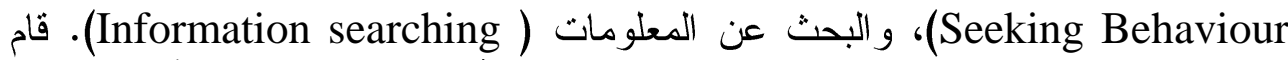

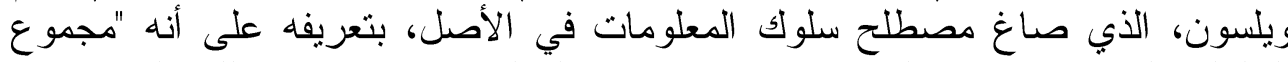

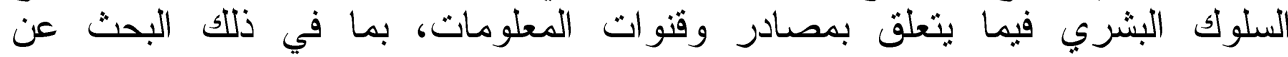

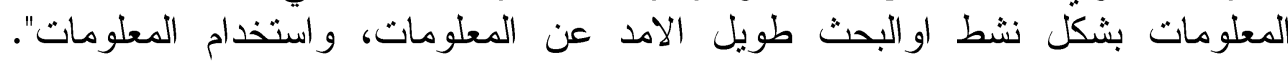

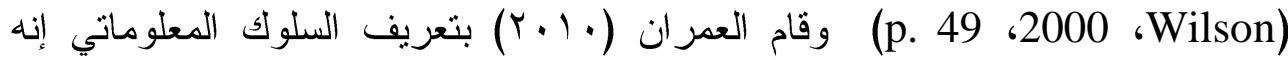

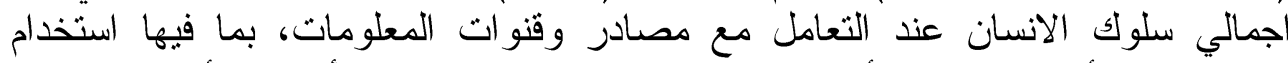

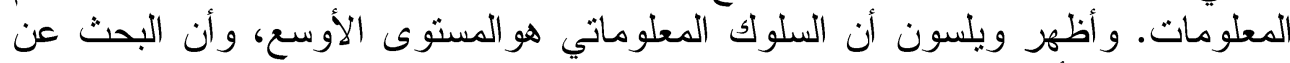

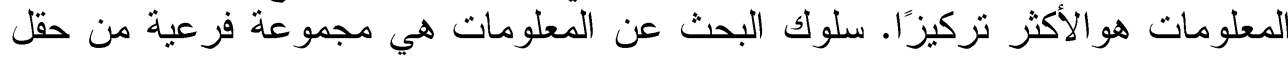

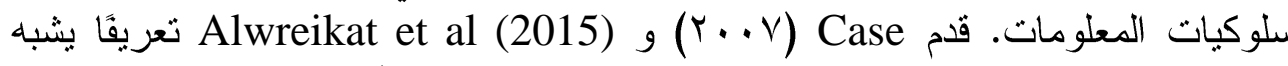

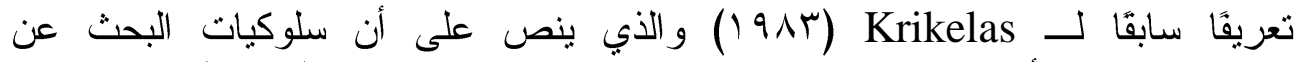

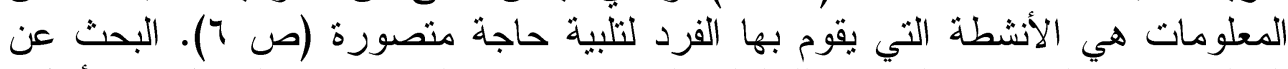

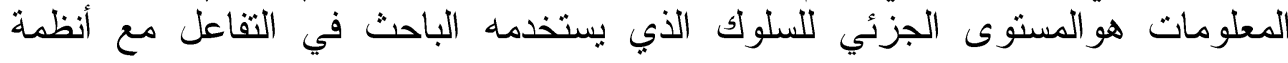

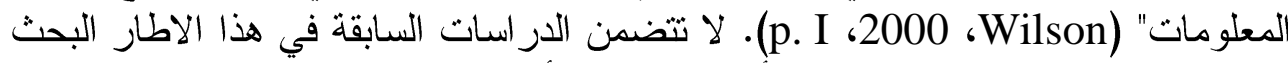

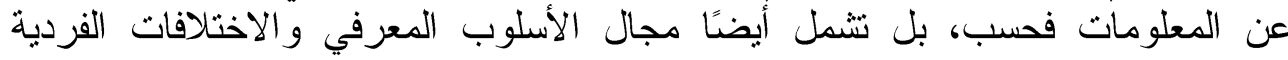

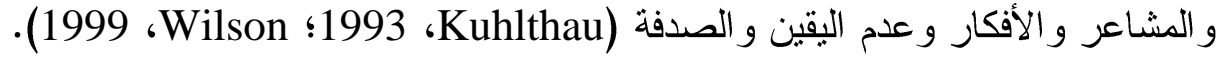

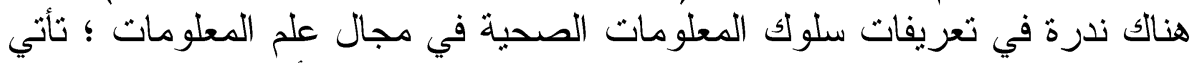

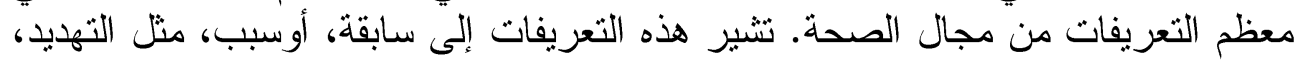

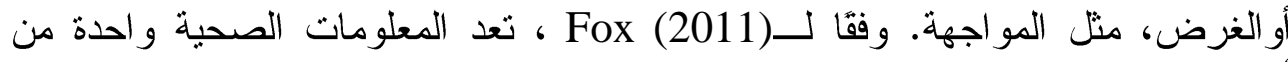

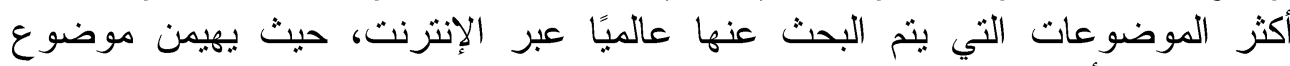

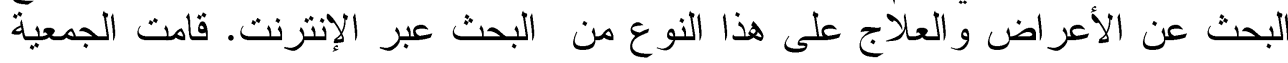

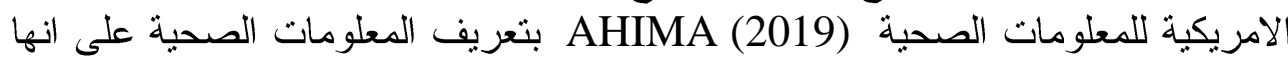




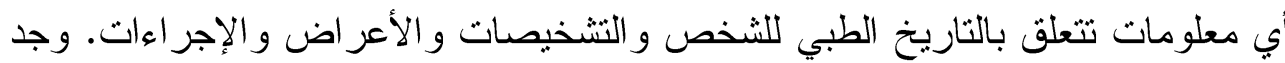
Renahy and Chauvin

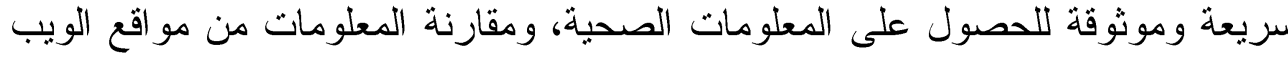

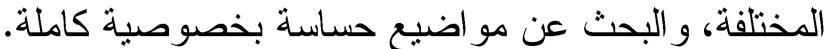

هناك العديد من الدراسات المتعلقة بالمعلومات الصحية التي ثركزة كلئ على كيفية

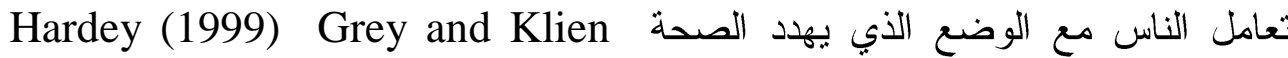

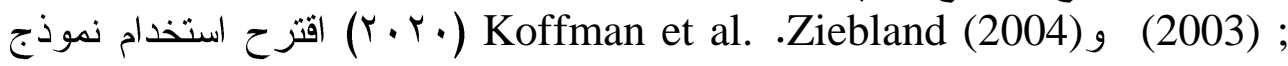

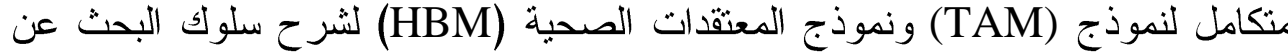

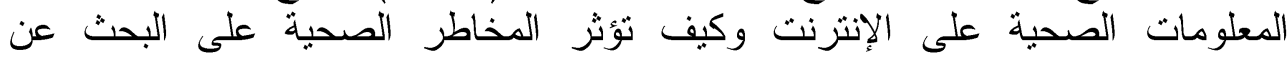

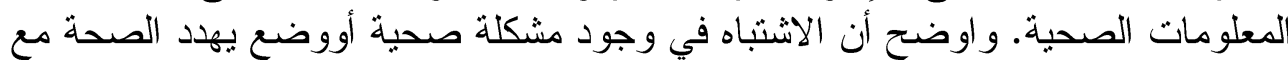
نقص المعلومات قد يؤدي إلى الشعور بعدم اليقين. وهذا بدوره بعان يعمل على امكانية

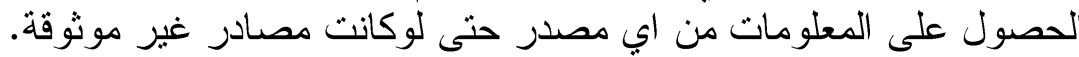

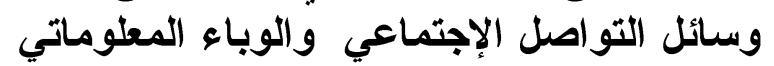

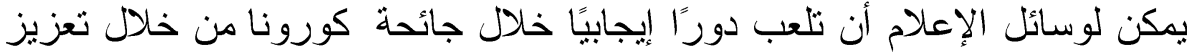

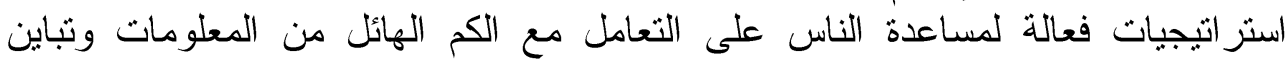

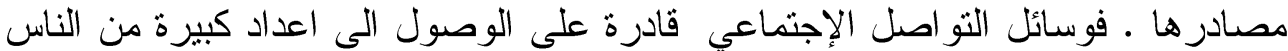

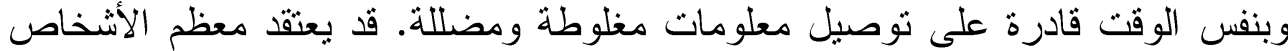

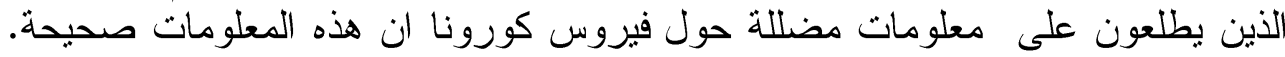
ويميل العديد من مستخدمي الثبكات الإجنماعية إلى تبادل المعلومات الثين الخاطئة و إثارة الخوف من شيء ليس بالضرورة صحيحًا عن طريق النشرخدع عبر وسائل الإعلام.

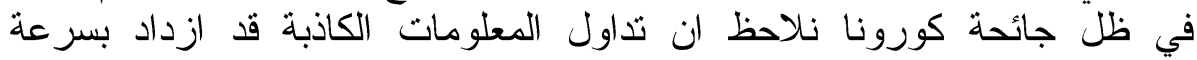
Koffman et al.

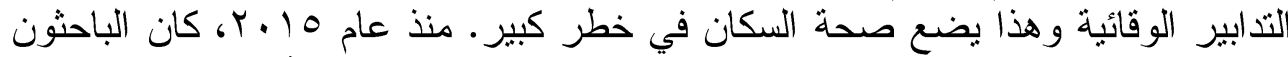

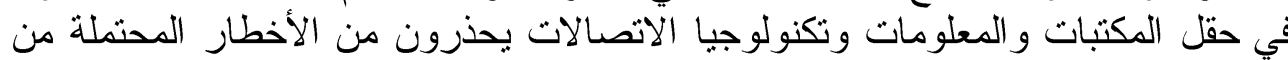

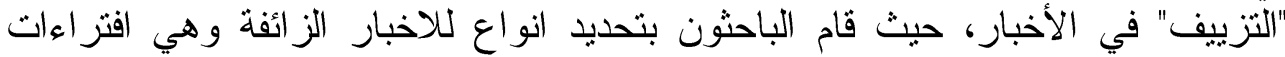

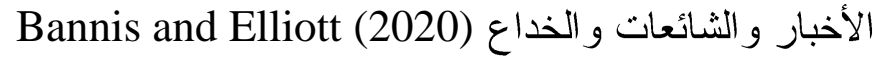

يمكن تفسير ما كان بُطلق عليه غالبًا بشكل عشو ائي "مشكلة الأخبار الزائفة الئة بشكل

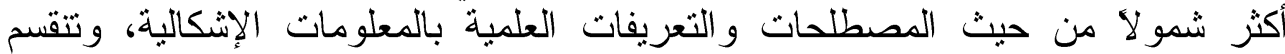

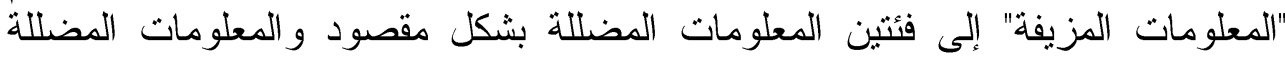

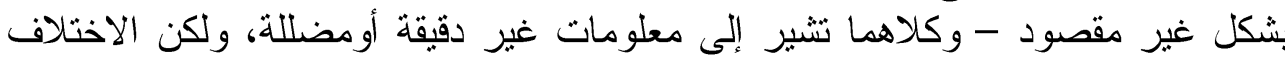

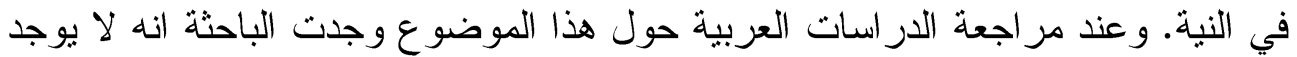

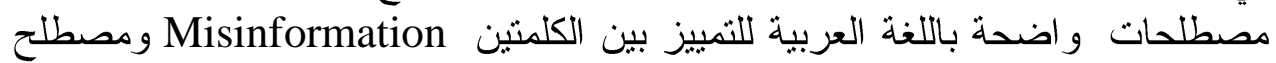

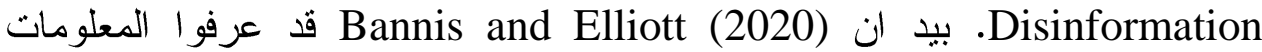
المضللة غير المقصودة بانها معلومات غير مقصودة وتتضمن أخطاء أوعدم دقةة، بينما

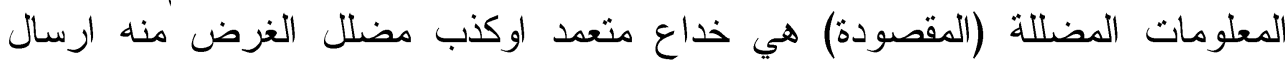

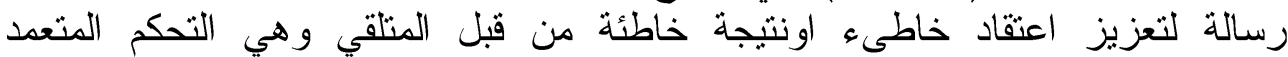
بالمعلومات لانثاء اعتقاد خاطى أنى. 
غالبًا ما يكون السبب الرئيسي للأخبار المزيفة لتحقيق منافع مالية أومكاسب لئب

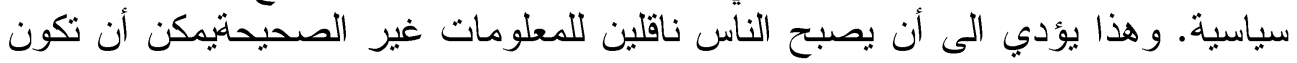

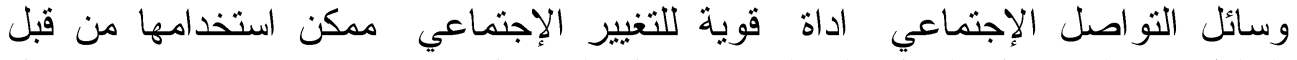

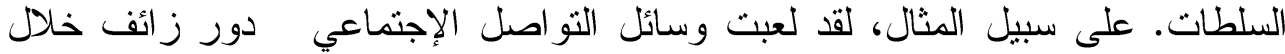

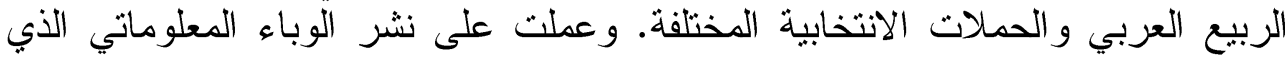

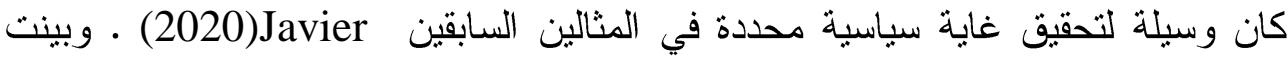

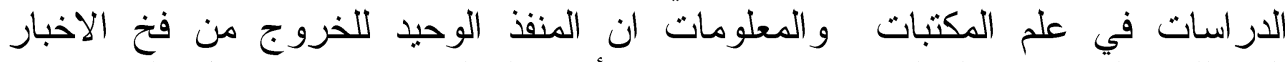

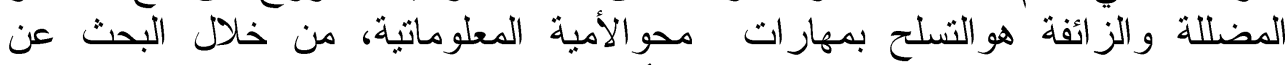

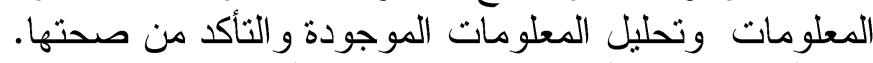

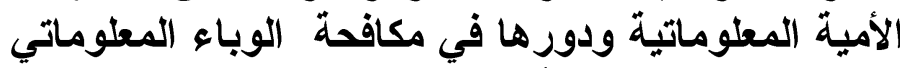

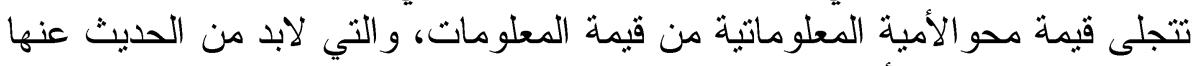

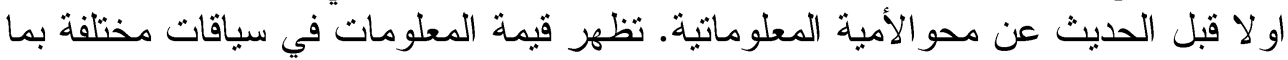

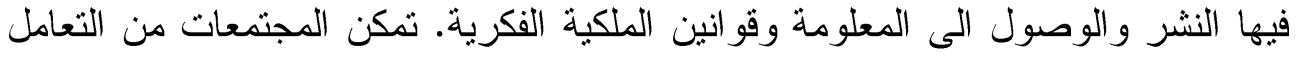

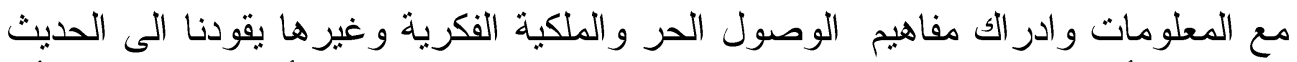

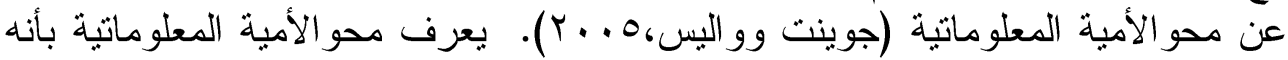

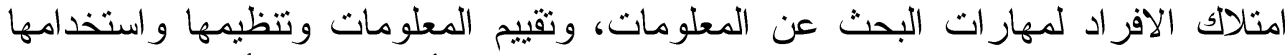

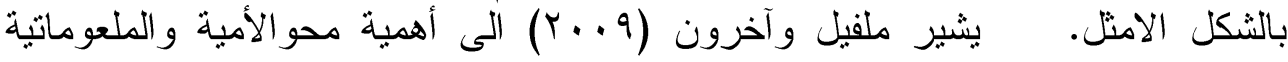

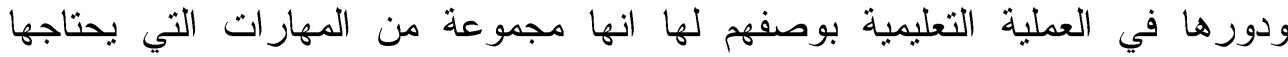

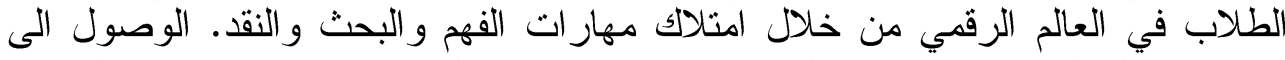

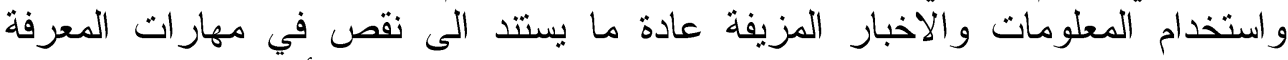

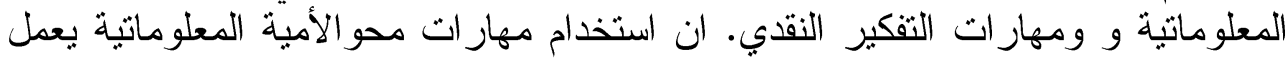

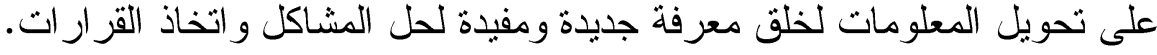

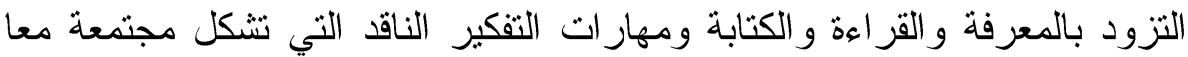

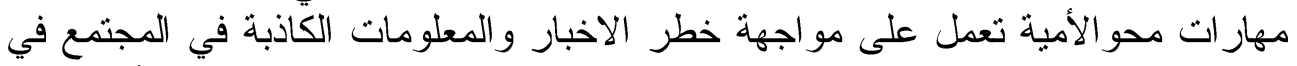

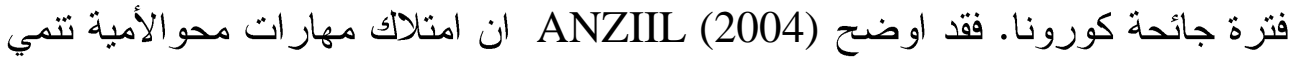

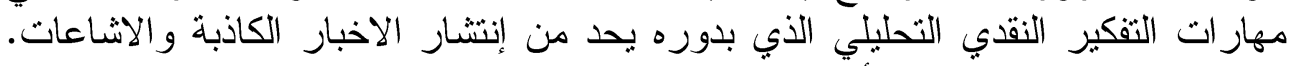
يمكن للشخص المتعلم ان يتأكد من موثوقية وصحة للفي المعلومات من خلال وضل وضع معيار

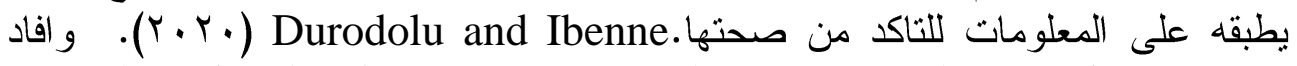

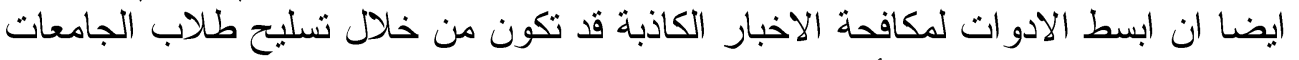

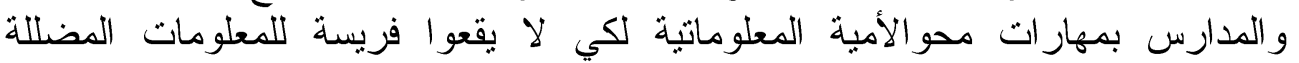
و الاخبار الكاذبة.

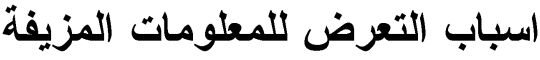

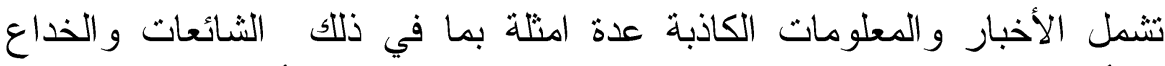

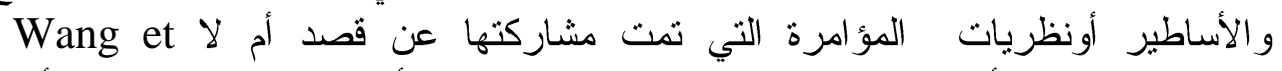
( ب l 9al)

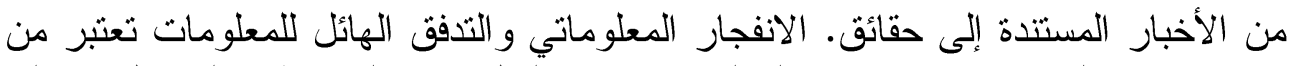

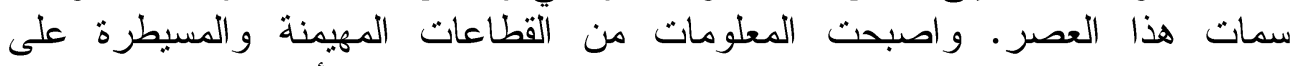

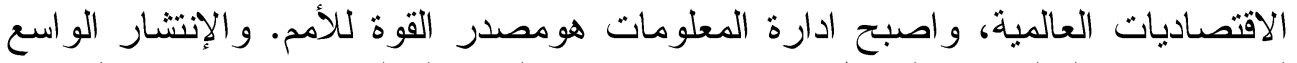

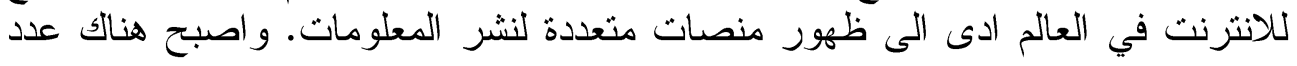


مبير من المنصات التي تتشر معلومات مضللة. و وعادة ما يكون البشر غير قادرين تمامًا

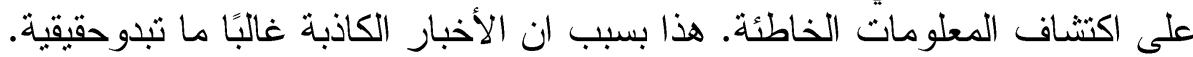

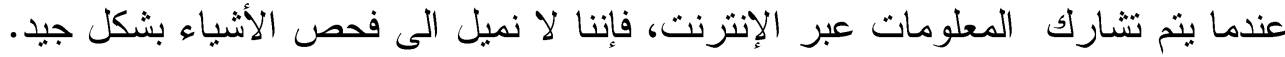

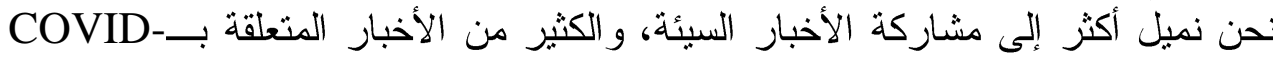

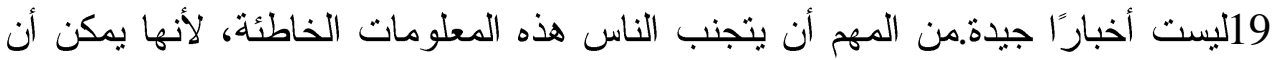

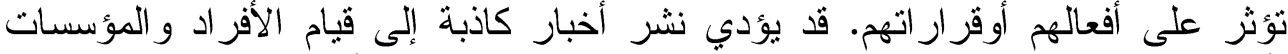

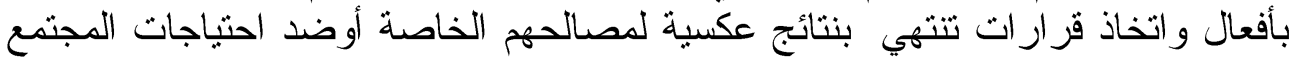
ويمكن أن يكون لها عو اقب وخيمة على الصحة العامة (Adebimpe et al, 2015).

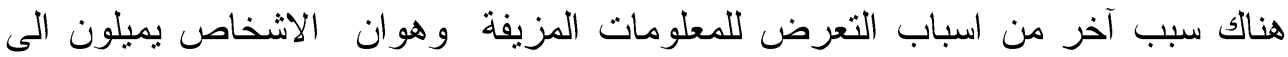

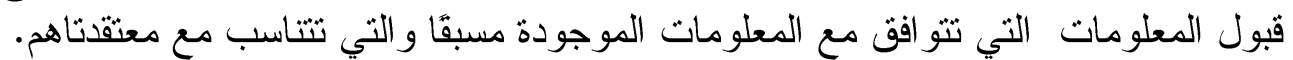
امثلة على الاخبار و المعلومات التز ائفة من وسائل التو اصل الصل الإجتماعي

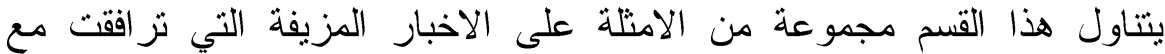

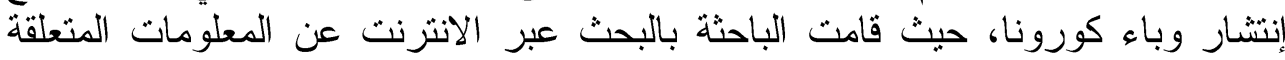

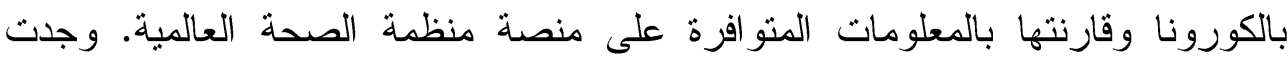

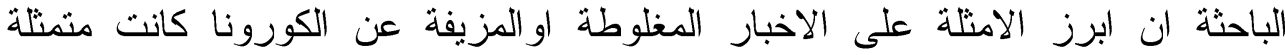

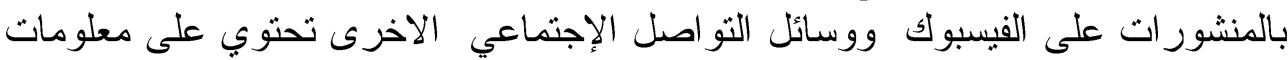

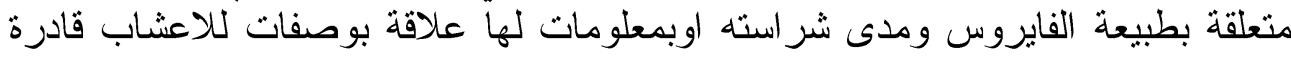

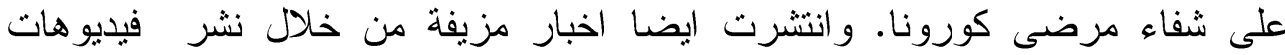

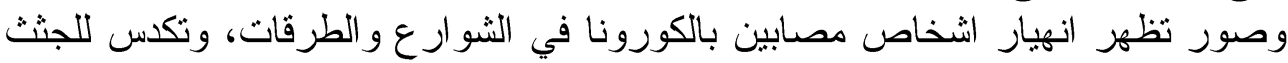

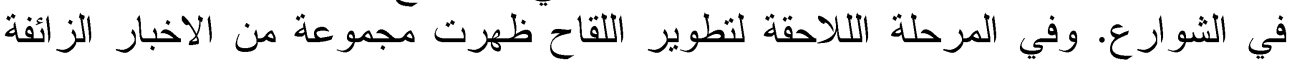

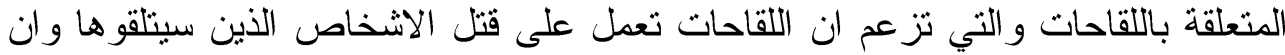

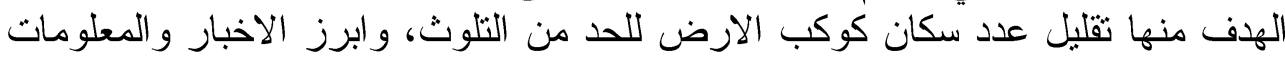

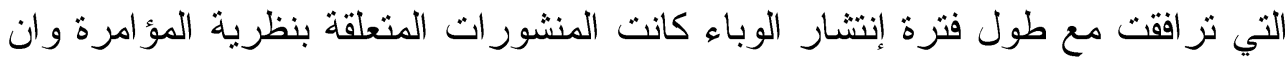

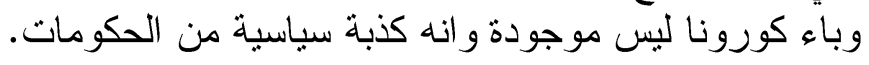

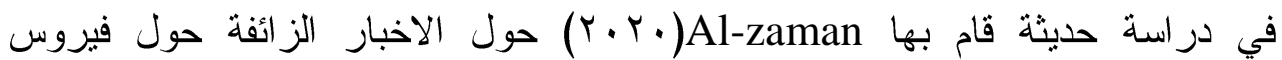

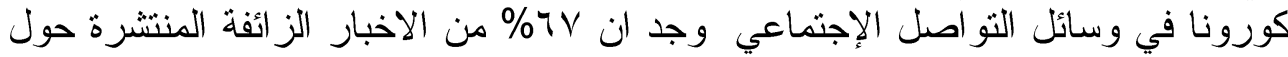

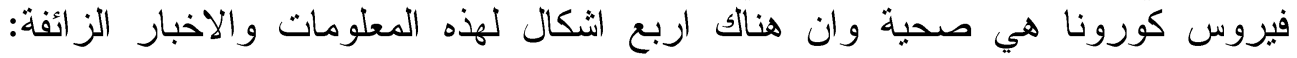

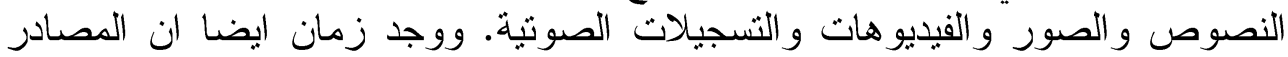

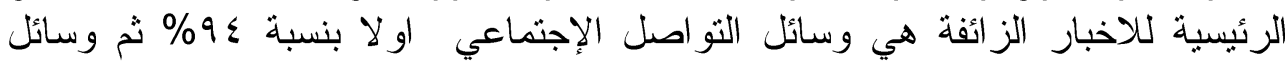
الاعلام ثانيا. ووجد ايضا ان ما نسبته ع7\% من الاخبار المتعلقة بكورونا كانت سلبية. مهار ات تحديد الاخبار الز ائفة لكي يكون الفرد قادرا على تتبع الاخبار و المعلومات الزائفة بجب عليه بداية

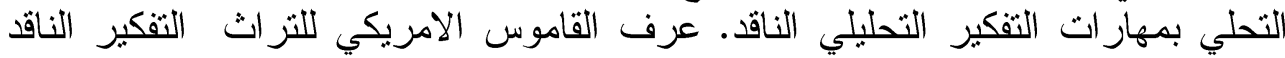

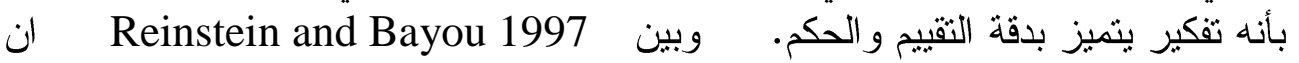

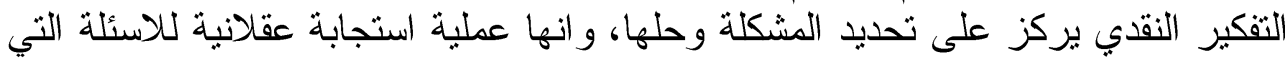
لا بمكن الاجابة عليها بشكل نهائي وغالبا تكون جميع المعلومات ذات الصلة غير متوفرة

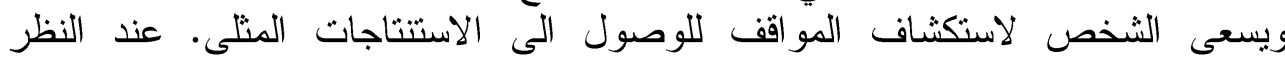

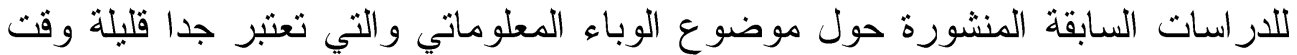


كتابة هذا البحث فان الباحثة وجدت ان هنابك مجموعة من البان المهارات التي من المككن

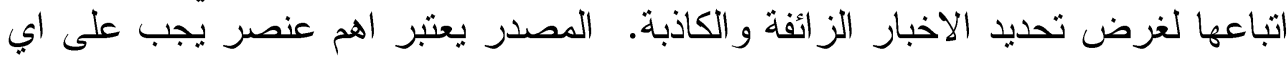

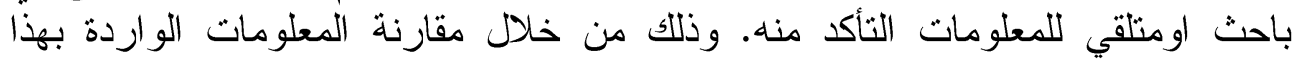
المصدر بالمعلومات الموجودة في المصادر الرسمبة مثل وكالات الانباء الرسمبة.

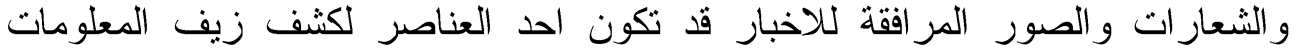

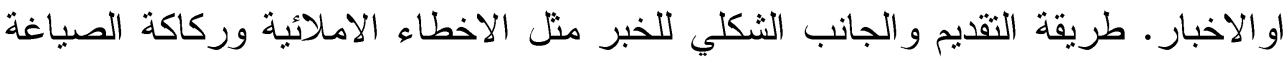

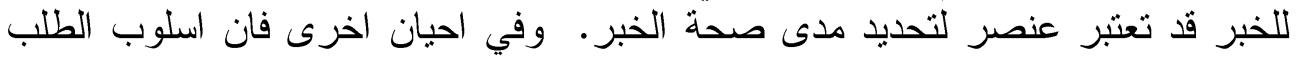

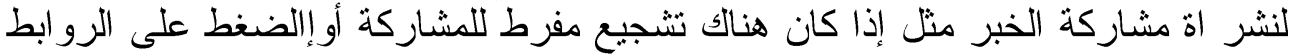

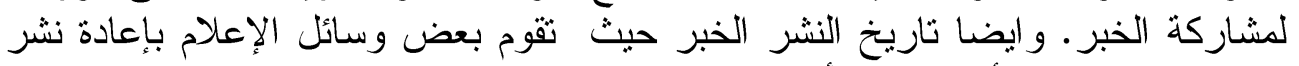

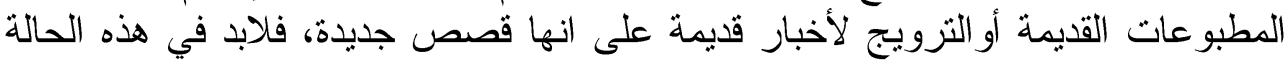

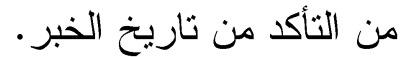

كل هذه المعلومات التي تز افقت مع جائحة كورونا دفعت الهيئات الدولية

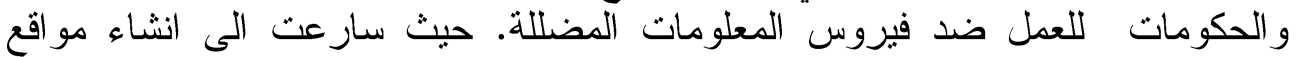

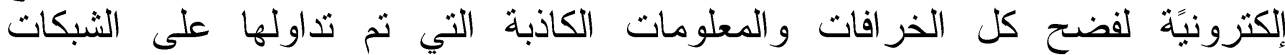

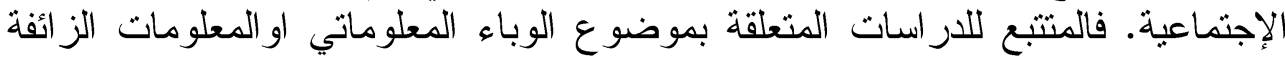

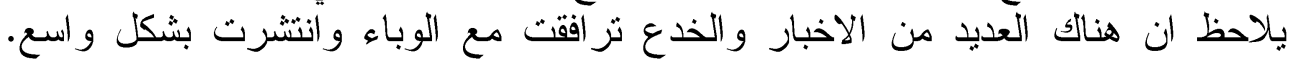

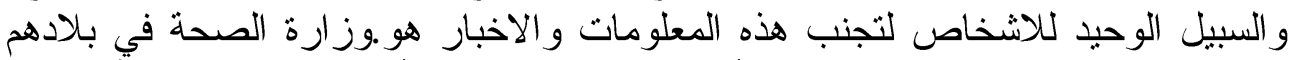

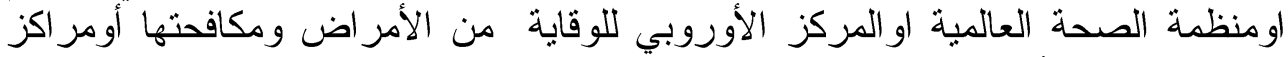

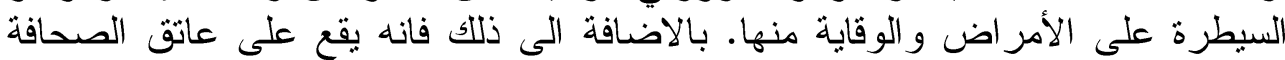

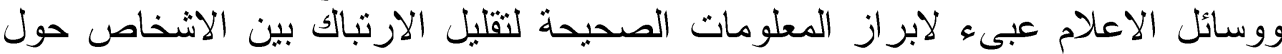

\section{الخاتمة والتوصيات}

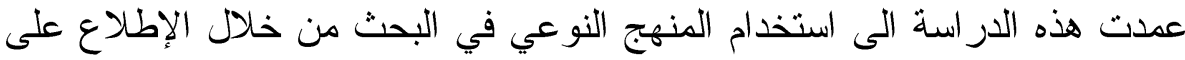

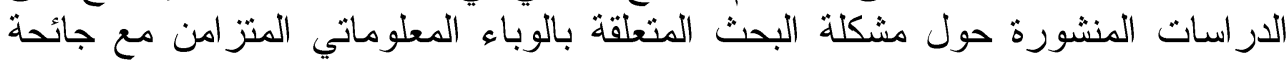

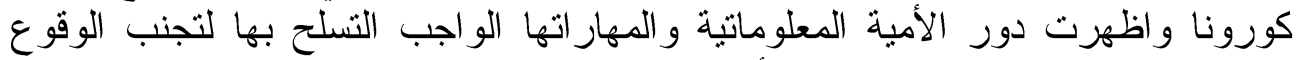

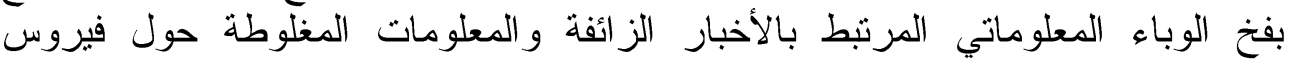

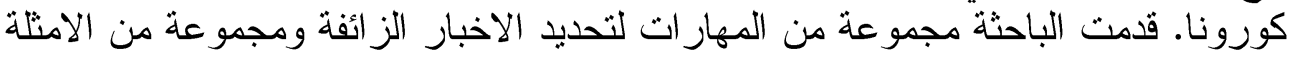

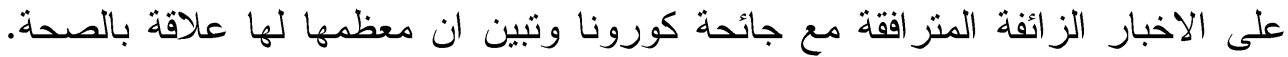

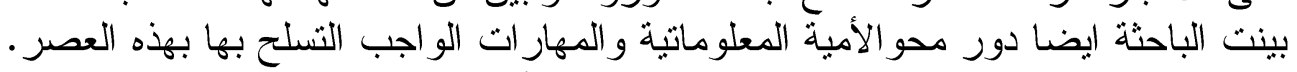
تقدم هذه الدراسة مجموعة من التوصيات ملخصة التصة بالآتي:

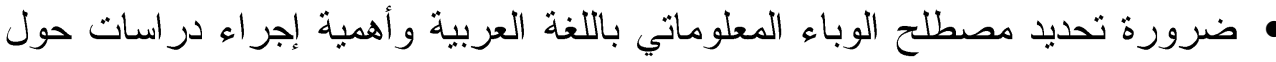
هذا الموضوع. • تحديد أهمية محو الأمية المعلوماتية ودور ها في الحد من ظاهرة الوباء المعلوماتي من

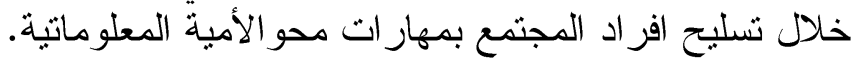

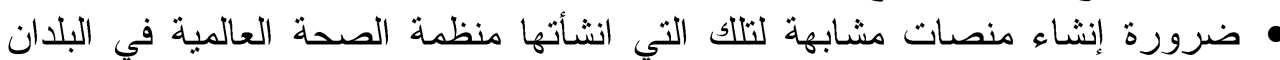

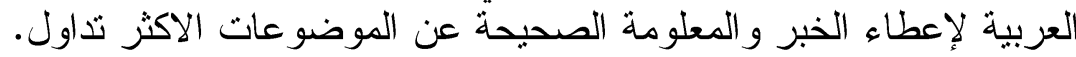

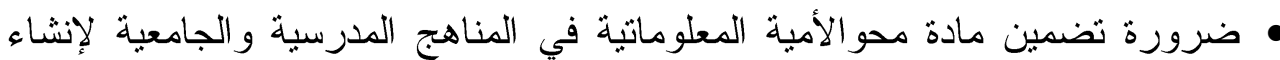

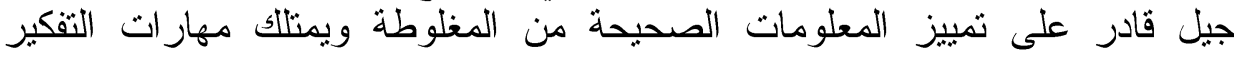




\section{Abstract \\ Information illiteracy and the information epidemic in light of the Corona pandemic Causes and ways to curb this epidemic By Asmaa El-Warikat}

The wide spread of social media led to an increase in sharing information and news. Health organizations are depending more on social media in sharing information and news. Corona virus (Covid-19) was declared as a pandemic by World Health Organization (WHO) and this lead an increase in the information and news load on the virus. This led to the existence of fake news and disinformation. WHO has declared as well the infodemic in concordance with the pandemic because of the huge amount of fake news accompanied the spread of Corona virus. This study aims to investigate the infodemic and the role of information literacy in avoiding the infodemic. The researcher used content analysis of research available on the topic, the study presented examples on the fake news and disinformation on the topic. At the end the researcher presented a group of skills to identify fake news and recommendations.

Keywords: Infodemic, information seeking behavior, Disinformation, Information literacy, Corona Virus (Covid-19).

\section{References}

Adebimpe W.O., Adeyemi D.H., Faremi A., Ojo J.O., Efuntoye A.E. (2015) The relevance of the social networking media in Ebola virus disease prevention and control in Southwestern Nigeria. Pan Afr. Med. J.;22(Suppl. 1) doi: 10.11694/pamj.supp.2015.22.1.6165. - DOI - PMC - PubMed

AHIMA (2019). Health Data and Information Conference. Available at: https://www.emedevents.com/c/medical-conferences-2019/american-health-informationmanagement-association-ahima-convention-exhibit-2019

ANZIL (2004) Australian and New Zealand Institute for Information Literacy (ANZIIL) Available at: https://www.utas.edu.au/_data/assets/pdf_file/0003/79068/anz-info-litpolicy.pdf

Al-Wreikat, A., Rafferty, P. and Foster, A. (2015), "Cross-language information seeking behaviour English vs Arabic", Library Review, Vol. 64 No. 6/7, pp. 446467. https://doi.org/10.1108/LR-04-2015-0044

Al-Zaman, Sayeed (2020) in-print, "COVID-19-related Fake News in Social Media", Health science available at: https://www.medrxiv.org/content/10.1101/2020.07.06.20147066v1

Bannis, Darrin and Elliott Robert (2020) Defning misinformation, disinformation and malinformation: An urgent need for clarity during the COVID-19 infodemic. Available at: https://www.researchgate.net/profile/Darrin_Baines/publication/341130695_Defining_misi nformation disinformation and malinformation An urgent need for clarity during the COVID-19 infodemic/links/5eb01d1b299bf18b9594b28f/Defining-misinformationdisinformation-and-malinformation-An-urgent-need-for-clarity-during-the-COVID-19infodemic.pdf?origin=publication_detail

Fox, Susannah,( 2011), The Social Life of Health Information available, Internet and Technology at: https://www.pewresearch.org/internet/2011/05/12/the-social-life-of-healthinformation-2011 
Hardey M. Doctor in the house (1999): The Internet as a source of lay health knowledge and the challenge to expertise. Sociol Health Illn 21: 820-35.

Gray NJ, Klein JD, Sesselberg TS, Cantrill JA, Noyce PR (2003). Adolescents' health literacy and the Internet. J Adolesc

Health32: 124

Javier Cifuentes-Faura (2020) “ Infodemics during COVID-19: resources and recommendations to combat it" Online Information Review Vol.ahead-of-print No. aheadof-print. https://doi.org/10.1108/OIR-08-2020-0352

Koffman et al (2020)Uncertainty and COVID-19: how are we to respond? Journal of the Royal Society of Medicine Vol 113, No 6 available at: https://www.ncbi.nlm.nih.gov/pmc/articles/PMC7439590

KRIKELAS, J. (1983). Information-seeking behavior: Patterns and concepts. Drexel library quarterly, 19, 5-20.

KUHLTHAU, C. C. (1993). A principle of uncertainty for information seeking. Journal of documentation, 49, 339-355.

Mark Hepworth and Geoff Walton (2013), "Developing People’s Information Capabilities: Fostering Information Literacy in Educational, Workplace and Community Contexts", Developing People's Information Capabilities: Fostering Information Literacy in Educational, Workplace and Community Contexts (Library and Information Science, Vol. 8), Emerald Group Publishing Limited, p. i. https://doi.org/10.1108/S1876$\underline{0562(2013) 0000008021}$

Melville, D., et al. (2009). Higher education in a Web2.0 world: Report of an independent committee of inquiry into the impact on higher education of students' widespread use of Web 2.0 technologies. [Online]. Retrieved from http:// www.jisc.ac.uk/media/documents/publications/heweb20rptv1.pdf. Accessed on 10 November 2011

Oluwole Olumide Durodolu and Samuel Kelechukwu.(2020), "The fake news infodemic vs information literacy” LIBRARY HI TECH NEWS, No 7, pp13-14.

Nicholas Joint and Jake Wallis. (2005), Information literacy and the role of national library and information associations Library Review, vol. 54 no. 4

59: $1783-93$

The Association of College and Research Libraries (2016) Framework for Information Literacy for Higher Education booklet is available for download at: http://www.ala.org/acrl/files/issues/infolit/framework.pdf

PAHO (2020) available at:https://iris.paho.org/bitstream/handle/10665.2/52052/Factsheetinfodemic_eng.pdf?sequence $=14 \&$ isAllowed=y

Reinstein, A. and Bayou, M.E. (1997), "Critical thinking in accounting education: processes, skills and applications", Managerial Auditing Journal, Vol. 12 No. 7, pp. 336342. http://0a1014c53.y.https.doi.org.bau.proxy.coe-

elibrary.com/10.1108/02686909710180698

Renahy, E., Parizot, I. \& Chauvin, P. Health information seeking on the Internet: a double divide? Results from a representative survey in the Paris metropolitan area, France, 20052006. BMC Public Health 8, 69 (2008). https://doi.org/10.1186/1471-2458-8-69

Wang Y, McKee M, Torbica A, Stuckler D. Systematic Literature Review on the Spread of Health-related Misinformation on Social Media. Soc Sci Med. 2019 Nov;240:112552. doi: 10.1016/j.socscimed.2019.112552. Epub 2019 Sep 18. PMID: 31561111; PMCID: PMC7117034

WILSON, T. D. 2000. Human information behavior. Informing science, 3, 49-56

Ziebland S. (2004) The importance of being expert: the quest for cancer information on the Internet. Soc Sci Med 